\title{
Kritik an den Empfehlungen der EKAF zur Prävention HIV-Infizierter unter ART
}

\author{
AIDS-Aufklärung Schweiz \\ $(A A S)^{*}$
}

\section{* Dr. med. Kurt April, Horgen; Dr. med. Alessandro Caponi, Lausanne; Prof. Dr. med. Marcel Capt, Les Charbonnières; Dr. med. Giovanni Fantacci, Niederhasli; Prof. Dr. med. Max Geiser, Wabern; Dr. med. Ralph Kaiser, Zürich; Dr. med. Thomas Keller, Pfäffikon SZ; Prof. Dr. med. Hans Koblet, Burgdorf; Dr. med. Eric Rochat, Saint-Légier; RA lic. iur. Ilona Singer, Zürich; Dr. med. Wolfgang Steinke, Schaffhausen Dr. med. Dieter Walch, Vaduz}

Interessenverbindungen: keine

Replik der EKAF auf Seite 1304.
Dass HIV-infizierte Menschen unter ART deutlich weniger infektiös sind, ist erfreulich. Allerdings erachtet es die AAS - angesichts der wissenschaftlichen Fakten - zurzeit als unangebracht, davon auszugehen, HIV-infizierte Menschen unter ART seien nicht infektiös. Auch wenn zukünftig auf diese Weise «nur» einzelne Ansteckungen stattfinden sollten, sind diese zuviel. Die AAS lehnt eine Änderung der Rechtsprechung ab, bei der HIV-infizierte Personen unter ART, die keine Präventionsvorschriften beachten, von einer Strafe freigesprochen werden sollen, denn aus Sicht der Prävention würde sich dies kontraproduktiv auswirken.

Zusätzlich bedeuten die Empfehlungen der Eidgenössischen Kommission für Aidsfragen (EKAF) ein irreführendes Signal an die Bevölkerung, weil diese als eine Entwarnung vor der Gefahr der HIV-Epidemie aufgefasst werden könnten. Die AAS schliesst sich damit der weltweiten Kritik der Experten an den EKAF-Empfehlungen an. Ein Alleingang der Schweiz ist nicht angebracht.

\section{HIV - das raffinierte Virus}

Die HIV-Infektion ist seit 25 Jahren bekannt. In dieser Zeit wurden unvergleichlich hohe Investitionen in Forschung, Behandlung und Prävention getätigt, um dieser fast immer tödlich verlaufenden Infektionskrankheit Einhalt zu gebieten. Das Lentivirus HIV ist raffinierter als andere Viren oder Bakterien. Noch heute sind wir weit von der Entwicklung einer Impfung entfernt. Die Anzahl HIV-infizierter Menschen steigt weiterhin weltweit, nach Schätzung der WHO [1] auch in Europa und Nordamerika.

Einer der bisher grössten Erfolge in der Bekämpfung der HIV-Epidemie war die Einführung der antiretroviralen Therapie (ART), die die HIVInfektion von einer tödlich verlaufenden Infektionskrankheit in eine chronische überführen konnte. Damit konnte die Aidserkrankungs- und sterberate in den westlichen Ländern deutlich gesenkt werden. Das Leben von HIV-infizierten Personen kann verlängert und ihre Lebensqualität deutlich verbessert werden.

Dennoch bleibt die HIV-Infektion bis heute unheilbar. ART vermag zwar die Konzentration der HI-Viren im Blut (Viruslast) unter die Nach- weisgrenze zu senken, doch schon wenige Tage oder Wochen nach Absetzen der Therapie kommt es zum raschen Anstieg der Viruslast im Blut [2].

\section{Verminderte Infektiosität unter ART}

Parallel zur Viruslast im Blut verhält sich auch die Viruslast in den Genitalsekreten. Deshalb sinkt durch ART auch die Infektiosität bei allen Übertragungswegen stark (sexuell, von Mutter auf Kind, durch Blut). In einigen Studien mit serodifferenten Paaren, bei denen der HIV-infizierte Partner unter ART stand, konnte keine HIV-Übertragung festgestellt werden [3].

\section{Stellungnahme der EKAF \\ zur HIV-Infektiosität und -Prävention}

Die Eidgenössische Kommission für Aidsfragen (EKAF) behauptet nun in ihrer Stellungnahme [4], die Infektiosität bei HIV-infizierten Menschen unter ART mit vollständig unterdrückter Virämie sei so gering, dass die Gefahr einer HIVÜbertragung vernachlässigt werden könne. Sie seien sexuell und von Mutter zu Kind nicht mehr infektiös.

Aus diesem Grunde könne eine solche Person auf präventive Massnahmen wie Kondomanwendung verzichten und mache sich folglich auch nicht mehr strafbar, wenn sie ungeschützt sexuell aktiv sei.

Den Verzicht auf Kondomanwendung will die EKAF auf serodifferente Paare (nur ein Partner ist infiziert) unter folgenden Bedingungen (im folgenden als «HIV-infizierte Menschen unter wirksamer ART» bezeichnet) beschränken: 1. ART wird durch den HIV-infizierten Menschen eingehalten und durch den behandelnden Arzt kontrolliert; 2. die Viruslast liegt seit mindestens sechs Monaten unter der Nachweisgrenze; 3. es besteht keine Infektion mit anderen sexuell übertragbaren Erregern (STD).

\section{Breites Medienecho und weltweite Kritik von Experten}

Die Stellungnahme der EKAF rief im In- und Ausland ein grosses Medienecho hervor. Es entstand der Eindruck, alle HIV-infizierten Menschen seien wirksam therapiert, hätten eine normale Lebensqualität und -erwartung und seien nicht mehr 
infektiös, kurz, die EKAF habe eine Entwarnung für die Gefahren der HIV-Epidemie gegeben.

Ein Beispiel, wie die EKAF auf diese Weise interpretiert wurde, liefert die sonst ausgewogen und kritisch berichtende Neue Zürcher Zeitung in ihrem Kommentar «Neue Lage - neue Rechtsprechung»: Der Kommentator fordert zur Änderung der bisherigen Rechtsprechung gegenüber allen HIV-Infizierten auf, die sich einer Therapie unterziehen. Darüber hinaus diskutiert er einen Verzicht der Verurteilung von «HIV-Positiven, die sich nicht einer Therapie unterstellen, über ihren Status nicht informieren und dennoch ungeschützten Sexualkontakt pflegen» [5].

Viele Gesundheitsbehörden wie die WHO [6], die amerikanischen Gesundheitsbehörden (CDC) [7], die Gesundheitskommission der EU und der französische nationale Aidsrat (Conseil national du sida, CNS) [8] distanzieren sich von der EKAF und warnen, man dürfe mit Studien, wie sie die EKAF aufführte, nicht die falsche Botschaft verbreiten, dass Aids eine weniger bedeutsame Krankheit geworden sei. Sie betonen, es gebe auch bei HIV-infizierten Personen unter wirksamer Behandlung keine hundertprozentige Sicherheit, weshalb keine Änderung der Prävention angezeigt sei. Alle sexuell aktiven HIV-infizierten Menschen sollten bei jeder sexuellen Aktivität und bei jedem Sexualpartner immer und korrekt Kondome anwenden.

\section{Die Infektiosität und Übertragungsrate von HIV unter ART}

Zum heutigen Zeitpunkt bestehen noch viele offene Fragen, unklare und widersprüchliche Befunde, die bezüglich der Infektiosität zur Vorsicht mahnen:

1. Bei einigen HIV-Infizierten unter wirksamer ART mit einer Viruslast unter der Nachweisgrenze konnten doch in mehreren Untersuchungen zellassoziierte Virusgenome in Genitalsekreten nachgewiesen werden $[9,10]$.

2. Erwirbt ein Patient unter wirksamer ART eine andere sexuell übertragbare Krankheit, steigt die Viruslast in Genitalsekreten und im Blut, wenn auch geringer als ohne wirksame ART $[11,12]$. Auch bei anderen entzündlichen Prozessen der Genitalien ist dies möglich. Diese Befunde stützen ebenfalls die Hypothese, dass zellassoziierte Genome, die auch unter ART gefunden werden, potentiell infektiös sein können.

3. ART konnte die Mutter-Kind-Übertragung effektiv senken [13, 14], aber nicht immer verhindern, wie die EKAF suggeriert. Bei einigen Studien stellte man trotz wirksamer ART einzelne HIV-Übertragungen fest [15]. Transiente Episoden von viraler Replikation und Mikrotraumen in der Plazenta werden als Ursache vermutet [16].

4. Wegen der Variabilität des HIV folgt unausweichlich mit der weltweiten Verbreitung von ART eine zunehmende Übertragung medikamentenresistenter HIV [17].

Schon zu oft wurde die Raffinesse des HI-Virus unterschätzt, weshalb die AIDS-Aufklärung Schweiz (AAS) mahnt, die angesprochenen Probleme ernst zu nehmen und nicht vorzeitig bezüglich HIV-Transmission Entwarnung zu geben.

Verschiedene Forscher ziehen aus denselben Befunden ganz andere Schlüsse als die EKAF. So betonen z.B. Neely et al. [9], bei HIV-infizierten Menschen trotz wirksamer ART die Möglichkeit einer HIV-Ansteckung von der Mutter auf das Kind, den Sexualpartner oder die Verbreitung von resistenten Viren und fordern, die HIV-infizierten Menschen dementsprechend zu beraten.

\section{Ärztliche Beratung: \\ kontraproduktive Vorschläge der EKAF}

Die AAS erachtet es als unabdingbar, HIV-infizierte Menschen unter ART sowie ihre Partner genau und individuell über die Übertragungsrisiken zu informieren. Auch kleine Risiken sollen besprochen werden.

Deshalb kritisiert die AAS die Aufforderung der EKAF an die behandelnden Ärzte, HIV-infizierte Menschen unter wirksamer ART - unabhängig davon, ob sie in einer festen Partnerschaft leben - zu informieren, dass sie nicht infektiös seien. Die EKAF begründet diese Empfehlung damit, dass diese Information entlastend wirken könne, da das Sexualleben von HIV-infizierten Menschen infolge von Infektionsängsten beeinträchtigt sei.

Richtig ist, dass die natürliche Hemmschwelle vor der HIV-Übertragung auf den Partner, aber auch die Angst vor einer Verurteilung wegen der Weiterverbreitung von HIV zu einer Sexualstörung führen kann $[18,19]$. Nur: Ist hier eine berechtigte Angst nicht ein guter Ratgeber? Ist eine Verminderung des sexuellen Verlangens nicht auch Ausdruck von Mitgefühl und Respekt vor dem Mitmenschen oder Partner? Viele HIV-infizierte Menschen möchten - gerade vor dem Hintergrund ihrer eigenen Erfahrungen mit der HIV-Infektion - ihre Mitmenschen vor einer HIVAnsteckung verschonen. Sie möchten nicht, dass ihre Partner oder Kinder dasselbe Schicksal durchmachen müssen wie sie. Aufgabe von uns Ärzten ist es, HIV-infizierte Menschen darin zu unter- 
stützen, ihr Leben lebenswert zu gestalten und ihnen zu helfen, trotz eines schlimmen Schicksalsschlags ihre Mitmenschlichkeit zu bewahren. Die Empfehlung der EKAF für die ärztliche Beratung läuft der ärztlichen Ethik entgegen.

\section{Keine Änderung der Rechtsprechung}

Die EKAF fordert, die Rechtsprechung für alle «HIV-Infizierte unter wirksamer ART» zu ändern, da sie sexuell nicht infektiös seien: Ein ungeschützter Sexualkontakt einer HIV-infizierten Person unter wirksamer ART mit einer HIVnegativen Person könne «weder den Tatbestand einer versuchten Verbreitung einer gefährlichen Krankheit im Sinne von Art. 231 Strafgesetzbuch noch den Tatbestand der versuchten gefährlichen Körperverletzung nach Art. 122, 123 oder 125 StGB erfüllen».

Wie oben dargelegt, ist nach heutigem Wissensstand keinesfalls sicher, dass ein HIVInfizierter unter wirksamer ART sexuell nicht infektiös ist.

Zudem wird ein HIV-infizierter Mensch unter wirksamer ART, der nicht in einer festen Partnerschaft lebt, kaum einsehen können, weshalb er seine(n) Sexualpartner über seine HIV-Infektion informieren soll und warum er Kondome anwenden soll. Und wie wird der HIV-infizierte Mensch denken und handeln, der ungenügend auf ART anspricht oder der die Medikamente unzuverlässig einnimmt? Und wie HIV-infizierte Menschen, die noch nicht mit ART behandelt werden, weil die CD4-Zellen nur wenig über $350 / \mathrm{mm}^{3}$ liegen, was eine geringe Infektiosität bedeutet? Es ist eine bekannte Tatsache, dass gerade im Bereich der Sexualität viele Menschen die Wirklichkeit nach ihren Wünschen zurechtbiegen.

Die AAS erachtet es als sehr wichtig, die geltende Rechtsprechung beizubehalten und die Bevölkerung mit Nachdruck darauf hinzuweisen. Mit ihrer Forderung unterläuft die EKAF die präventive Wirkung des Gesetzes.

\section{Irreführendes Public-Health-Signal an die Bevölkerung}

Die Safe-Sex-Botschaften der letzten 25 Jahre waren anerkanntermassen ungenügend, so dass neue innovative und effektive Public-HealthStrategien gefragt sind [20]. Die neuen Empfehlungen der EKAF gehören nicht dazu. Sie kommen einer Entwarnung vor der Gefahr der HIVInfektion gleich. Und dies in einem Jahrzehnt, in dem die Rate der Neuinfektionen nicht mehr zurückgeht, in einzelnen Untergruppen der Bevölkerung sogar ein Anstieg der HIV-Epidemie festgestellt werden muss [21, 22]. Alarmierend sind die Untersuchungen, die in der Schweiz [23] wie in anderen Industrieländern [24] nachweisen, dass 30-50\% der HIV-Infizierten erst kurz vor dem Auftreten von Aidssymptomen die Diagnose HIV-Infektion gestellt bekommen, so dass diese Menschen unwissentlich ihre Infektion weitergeben. Diese Fakten müssen als ein Public-Health-Versagen angesehen werden [20].

Darüber hinaus propagiert die EKAF im gleichen Artikel als einzige Präventionsbotschaft für die ganze Bevölkerung: «Eindringen immer mit Gummi, kein Sperma oder Blut in den Mund.» Diese Botschaft bringt die Präventionsphilosophie der postmodernen 80er Jahre zum Ausdruck, als eine Einschränkung der persönlichen Freiheit selbst durch eine tödliche Infektionskrankheit nicht sein durfte. Seither hat sich das Präventionsverständnis jedoch wesentlich geändert: Der Kondomgebrauch ist ein Teil eines umfassenderen Ansatzes [25] mit HIV-Test, Beratung, früher Behandlung von HIV-Infizierten mit ART, Treue, sorgfältiger Partnerwahl, Abstinenz sowie Partnerbenachrichtigung, postexpositioneller Prophylaxe usw.

\section{Um was es auch noch ging}

Der Sachkundige fragt sich, warum die EKAF Empfehlungen herausgibt, die die gesamte Fachwelt vor den Kopf stossen. In einem Interview in der Ars Medici vom 14. März 2008 [26] erläutert Prof. Vernazza, Präsident der EKAF, die Hintergründe, weshalb die EKAF ihren Artikel lancierte: «Wir wollen damit eine breite Diskussion anschieben, um die Entstigmatisierung von HIVPositiven zu fördern» und gleichzeitig die Rechtsprechung ändern, damit ungeschützter Sex von Menschen mit HIV kein Delikt mehr sei. Für HIV-Positive seien die EKAF-Empfehlungen eine riesige Erleichterung, weil sie dadurch gleichsam das Stigma verlieren würden, für andere eine gefährliche Infektionsquelle zu sein.

Verlor die EKAF wegen des Ziels «Entstigmatisierung» das Augenmass? Verwickelte sie sich deshalb in folgende Widersprüche? a) Die unterschiedlichen Empfehlungen beim Sexualverkehr für HIV-infizierte Menschen unter wirksamer ART mit und ohne feste Partnerschaft. Bei der Rechtsprechung sollen aber beide Gruppen wieder gleich behandelt werden. b) Im genannten Interview sagt Prof. Vernazza, es sei möglich, dass auch unter wirksamer ART Fälle von HIV-Übertragung vorkommen könnten. Im EKAF-Artikel hingegen heisst es unter den Titeln «Rechtsprechung» und «Bedeutung für HIV-infizierte Menschen», dass «HIV-infizierte Personen unter wirksamer ART nicht infektiös sind» und «HIV nicht weitergeben können». c) Um zu veranschaulichen, dass Vaginalverkehr eines HIV-infi- 
zierten Menschen unter ART ein vernachlässigbares Risiko aufweise, setzt die EKAF in ihrem Artikel dieses Risiko mit Küssen eines HIV-Infizierten gleich, wobei sie bei Küssen nicht einmal zwischen Zungen- und Wangenküssen differenziert. Im genannten Interview setzt nun Prof. Vernazza anstelle von Küssen den Oralverkehr als vergleichbares Risiko dar. Was gilt nun? Oralverkehr (bei dem Genitalsekrete auf die Mundschleimhaut gelangen) gilt in der Fachwelt als sexueller Übertragungsweg, wenn auch mit geringerem Risiko als Vaginalverkehr.

Im Interview lässt Prof. Vernazza auch durchblicken, dass sich die EKAF sehr wohl bewusst ist, dass sie andere Empfehlungen propagiert als der Rest der Welt. Nur: Das HI-Virus hält sich an keine Grenzen.

Die AAS ist der Meinung, dass die Bevölkerung ein Recht auf die ganze Wahrheit hat und keine beschönigende Darstellung braucht. Eine ideologisch-politische Motivation ist für HIVPräventionsempfehlungen ungeeignet.

Die EKAF befindet sich im Irrtum, wenn sie glaubt, durch ihre Empfehlungen die Stigmatisierung aus der Welt schaffen zu können. Für uns Ärzte ist es selbstverständlich, jeglicher Stigmatisierung und Diskriminierung entschieden entgegenzutreten. Widersprüchliche Aussagen von Ärzten und Behörden schaffen Verwirrung und fördern irrationales Verhalten der Bevölkerung wie z.B. die Diskriminierung von HIV-Infizierten.

\section{Literatur}

1 WHO Regional Office for Europe. Unpublished survey data on file. November 2007.

2 Bernasconi E, Vernazza PL, Bernasconi A, Hirschel B. HIV transmission after suspension of highly active antiretroviral therapy. J Acquir Immune Defic Syndr. 2001;27:209.

3 Castilla J, Del Romero J, Hernando V, Marincovich B, Garcia S, Rodriguez C. Effectiveness of highly active antiretroviral therapy in reducing heterosexual transmission of HIV. J Acquir Immune Defic Syndr. 2005;40:96-101.

4 Vernazza P, Hirschel B, Bernasconi E, Flepp M. HIVinfizierte Menschen ohne andere STD sind unter wirksamer antiretroviraler Therapie sexuell nicht infektiös. Schweiz Ärztezeitung. 2008;89(5):165-9.

5 Konsequent Therapierte sind nicht infektiös. NZZ vom 31.1.2008, S.15.

6 Sex ohne Kondom für gewisse HIV-Infizierte Schweizer Einschätzung gerät in die Kritik. SDA vom 1.2.2008, S.16.

7 Kurzstellungnahme vom 1.2.2008 der amerikanischen Gesundheitsbehörden. www.cdc.gov/ hiv/resources/press/020108.htm.

8 Einschätzung zu HIV-Infizierten in der Kritik. NZZ vom 2.2.2008.
9 Neely MN, Benning L, Xu J, et al. Cervical shedding of HIV-1 RNA among women with low levels of viremia while receiving highly active antiretroviral therapy. J Acquir Immune Defic Syndr. 2007;44: 38-42.

10 Kovacs A, Wasserman SS, Burns D, et al. Determinants of HIV-1 shedding in the genital tract of women. Lancet. 2001;358:1593-601.

11 Cohen MS, Hoffman IF, Royce RA, et al. Reduction of concentration of HIV-1 in semen after treatment of urethritis: implications for prevention of sexual transmission of HIV-1. Lancet. 1997;349:1868-73.

12 Sadiq ST, Taylor S, Kaye S, et al. The effects of antiretroviral therapy on HIV-1 RNA loads in seminal plasma in HIV-positive patients with and without urethritis. AIDS. 2002;16:219-25.

13 Garcia PM, Kalish LA, Pitt J, et al. Maternal levels of plasma human immunodeficiency virus type 1 RNA and the risk of perinatal transmission. N Eng J Med. 1999;431:394-402.

14 Rousseau C, Nduati R, Richardson B, et al. Longitudinal analysis of human immunodeficiency virustype 1 RNA in breast milk and of its relationship to infant infection and maternal disease. J Infect Dis. 2003;187:741-7.

15 Barreiro P, del Romero J, Leal M, et al. Natural preg nancies in HIV-serodiscordant couples receiving successful antiretroviral therapy. J Acquir Immune Defic Syndr. 2006;43:324-6.

16 Magder L, Mofenson L, Paul M, et al. Risk faktors and intrapartum transmission of HIV. J Acquir Immune Defic Syndr. 2005;38:87-95.

17 Cane P, Christie I, Dunn D, et al. Time trends in primary HIV drug resistance in the United Kingdom: observational study. BMJ. 2005;331:1368.

18 Kaplan HS. The Sexual Desire Disorders: Dysfunctional Regulation of Sexual Motivation. Levittown, PA: Brunner/Mazel; 1995.

19 Kaplan HS. The Real Truth About Women and AIDS How to Eliminate the Risks Without Giving Up Love and Sex. New York, NY: Simon \& Schuster; 1988.

20 Pillay D, Fisher M. Primary HIV infection, phylogenetics, and antiretroviral prevention. JID. 2007; 195:924-6.

21 Bundesamt für Gesundheit. HIV-Epidemie in der Schweiz 2007. BAG-Bulletin. 2008:28.84-8.

22 Murphy G, Chartlett A, Brown AE, Gill ON, Parry JV. Is HIV incidence increasing in homo/bisexual men attending GUM clinics in England, Wales and Northern Ireland? Commun Dis Public Health. 2004;7:11-4.

23 Bundesamt für Gesundheit. Keine weitere Abnahme neuer Aids-Fälle trotz wirksamer Therapien. BAGBulletin 2005;28:480-8.

24 CDC. Revised Recommendations for HIV Testing of Adults, Adolescents, and Pregnant Women in Health-Care Settings. MMWR. 2006;55(RR14);1-17.

25 Halperin DT, Steiner MJ, Cassell MM, et al. The time has come for common ground on preventing sexual transmission of HIV. Lancet. 2004;364:1913-5.

26 Ferber T. «Wir sind verpflichtet, unseren Patienten das ganze Wissen zu vermitteln.» Ein Gespräch mit dem Infektiologen und Aidsspezialisten Pietro Vernazza. Ars Medici. 2008;98(6):230-1. 